\title{
On the origin of the low energy cosmic rays from stellar sources*
}

\author{
S BISWAS, N DURGAPRASAD and SUSHMA S TRIVEDI \\ Tata Institute of Fundamental Research, Bombay 400005 , India
}

\begin{abstract}
The model of stellar origin of the anomalous component in the low energy cosmic rays for He to $\mathrm{Fe}$ ions observed in space vehicles is studied in the light of recent results. The model of heliospheric origin by Fisk et al which has several attractive features cannot explain the long-term variations of intensity observed during 1974 to 1978 as pointed out by Nagnshima and Morishita. The stellar origin model of Durgaprasad and Biswas, on the other hand, can easily account for the sudden appearance of the anomalous component in 1972 and its large decrease in intensity in 1978 on the basis of polarity reversal of the solar magnetic field as discussed by Nagashima and Morishita (1980). In this work, we show that in the stellar model energetic ions of $\mathrm{He}, \mathrm{C}, \mathrm{N}, \mathrm{O}$, etc. could originate in O-type stars which manifest very strong stellar wind with mass loss rate of $3 \cdot 10^{-6} \mathrm{M}_{\odot}$ per year. These have terminal velocities of about 1200 to $4000 \mathrm{~km} / \mathrm{sec}$ and are typically a few tines their escape velocity. These velocities correspond to ion energies of 10 to $100 \mathrm{keV} / \mathrm{amu}$. These ions are in partly ionised state and are accelerated in the interstellar shock fronts to about 1 to $50 \mathrm{MeV} / \mathrm{amu}$ and thus account for the observed anomalous component of low energy cosmic rays.
\end{abstract}

Keywords. Cosmic rays; anomalous component; stellar sources; Novae; O-type stars.

\section{Introduction}

In recent years a new component in low energy cosmic rays $(1-30 \mathrm{MeV} / \mathrm{amm})$ was discovered in the vicinity of earth and at distances upto $18 \mathrm{~A}$. U. (Hovestadt et al 1973; McDonald et al 1974; Garcia-Munoz et al 1975; Chan and Price 1974; Mewaldt et al 1975a; Webber et al 1975; Biswas et al 1975; Biswas and Durgaprasad 1980; and Webber 1979). The component has anomalous He, N, O and Ne abundances, that are quite different from solar and galactic cosmic rays. The IMP $7 \& 8$, Pioneer $10 \& 11$ and Voyager $1 \& 2$ spacecrafts have provided new and interesting information on the composition, time variation, radial and perpendicular gradient of this component. Pioneer 10 was at 20 A.U. in mid 1979 and travelling at the rate of 3 A.U. per year is likely to reach close to the heliospheric boundary at 50 A.U. by about 1985 . The data from these spacecrafts are expected to give additional information about this new component.

The origin of these particles is not definitely known at present. Several attempts have been made to explain its origin (Fisk et al 1974; Durgaprasad 1977; Durgaprasad and Biswas 1977; Biswas et al 1981). The model of Fisk et al (1974) have some attractive features which could partly account for the composition of the anomalous component. However, recent results show very large time variation of this component and the intensity reached very low value in early 1979 (Hovestadt et al 1979). Nagashima and Morishita $(1979,1980)$ discussed in detail the long-term modulation of the anomalous component and they pointed out that the heliospheric

Paper presented at the second national seminar-cum-workshop on the use and application of solid state nuclear track detectors held at Physical Research Laboratory, Ahmedabad, India, during 24-26 February 1981. 
model of Fisk et al (1974) cannot account for the long-term variations observed recently and these variations can be easily understood from the stellar model of Durgaprasad and Biswas (1977). They interpret the modulation as occurring due to the polarity reversal of the polar magnetic field of the Sun, one such reversal having occurred in the period of 1969-71. This interpretation is based on the hypothesis that when polar magnetic field of the Sun is nearly parallel to the galactic magnetic field, they both could easily connect with each other, and hence the low energy galactic cosmic rays could penetrate more easily into the heliosphere along the magnetic lines of force, as compared with those in the antiparallel state of the magnetic fields. This leads to a 22-year variation in cosmic ray intensity because polarity reversal occurs around every solar maximum. These authors point out that the sudden appearance of the anomalous component in 1972 and its absence in 1975 could be easily understood by the hypothesis of Durgaprasad and Biswas (1977) as a polarity reversal. Further, these authors point out that the observation presented by the Chicago group (McKibben et al 1979) that the anomalous component has abnormally large density gradient perpendicular to the solar equatorial plane can also be understood by the stellar origin model of Durgaprasad and Biswas (1977).

\section{Novae model of the origin}

Firstly, we discuss some of the significant aspects of the model of Durgaprasad and Biswas (1977) (Paper 1) as follows:

\subsection{Abundance of elements of some novae envelopes}

It is assumed that the sources of these particles are relatively close and particles emitted from novae explosion may be possible candidates. This was arrived at from some similarities in the abundances of $\mathrm{He}, \mathrm{C}, \mathrm{N}$ and $\mathrm{O}$ abundances in the anomalous

Table 1. Relative abundances in low energy cosmic rays and in some novae envelopes

\begin{tabular}{lccccc}
\hline Elements & $\begin{array}{c}\text { Low energy } \\
\text { cosmic rays } \\
\text { measured at } \\
\text { 10 MeV/amu } \\
\text { (a) }\end{array}$ & $\begin{array}{c}\text { Low energy } \\
\text { cosmic rays } \\
\text { demodulated } \\
\text { (b) }\end{array}$ & $\begin{array}{c}\text { Novae envelope } \\
\text { HR Del }\end{array}$ & $\begin{array}{c}\text { Galactic } \\
\text { cosmic rays } \\
(>1 \mathrm{GeV} / \mathrm{amu})\end{array}$ & Sun \\
\hline $\mathrm{He}$ & $60-200$ & $120-400$ & $150-700$ & 35 & $(\mathrm{c})$ \\
$\mathrm{C}$ & 1 & 1 & 1 & 1 & 180 \\
$\mathrm{~N}$ & 5 & 4 & 5 & 0.25 & 1 \\
$\mathrm{O}$ & 25 & 18 & 20 & 0.9 & 1.67 \\
$\mathrm{Ne}$ & 1 & 0.7 & - & 0.16 & 0.22 \\
$\mathrm{Mg}$ & 0.4 & 0.25 & 0.04 & 0.18 & 0.09 \\
$\mathrm{Si}$ & 0.5 & 0.3 & 0.04 & 0.12 & 0.10 \\
$\mathrm{Fe}$ & 0.1 & 0.1 & 0.05 & 0.1 & 0.08 \\
\hline
\end{tabular}

(a) Webber et al (1979)

(b) Durgaprasad and Biswas, (1977)

(c) Antipova, (1974)

(d) Webber et al (1972)

(e) See e.g. Biswas and Durgaprasad (1980). 
component and in some novae envelopes (table 1). It may be noted that because the spectral shapes of $\mathrm{He}, \mathrm{C}, \mathrm{N}, \mathrm{O}$, etc. for the anomalous component of the low energy cosmic rays are different from each other, the relative abundances are dependent on energy. For example, $\mathrm{O} / \mathrm{C}$ ratio is 4.4 and 4.7 at $9-30$ and $8-20 \mathrm{MeV} / \mathrm{amu}$ energy intervals respectively (McDonald et al1974; Biswas and Durgaprasad 1980), whereas at $10 \mathrm{MeV} / \mathrm{amu}$, the ratio is 25 (Webber 1979). The demodulated spectra and abundances are calculated using 'forced-field ' potential and the measured spectra at interplanetary space at a few A.U. It may also be noted that the measured abundances of some elements of some of the novae envelopes show some similarities whereas other elements such as $\mathrm{Mg}$, Si do not. After emission from the source during the propagation and acceleration stages the relative abundances of the ions will undergo changes which would depend on a number of parameters including the ionization states of the ions. The ion states are important properties of these particles as discussed in the following sections.

\subsection{Ionization states}

In this model it is assumed that ions are emitted from the source regions with energies of the order of $100 \mathrm{keV} / \mathrm{amu}$. At these energies the charged states of the ions, e.g. of carbon and oxygen are mostly $\mathrm{C}^{+1}, \mathrm{C}^{+2}$ and $\mathrm{O}^{+1}, \mathrm{O}^{+2}$, for equilibrium values of charge states in interstellar space.

The average charge, (i) carried by an ion moving through matter depends upon the atomic number $Z$ of the ion, its velocity, $v$, and on the nature of the medium. For velocity much greater than the electron velocity, the ion is fully stripped of its electrons and is completely ionised. For lower velocities and energies, it will retain some electrons and be partially ionised. In paper-1, calculations were made on the average charge $i$ carried by an ion of atomic number $Z$ and energy $E$ moving through interstellar space, which is assumed to consist mainly of hydrogen. In table 2 , we give the values of $i$ for various values of $Z(\mathrm{C}, \mathrm{N}, \mathrm{O}, \mathrm{Ne}, \mathrm{Mg}, \mathrm{Si}$ and $\mathrm{Fe}$ ions) and $E$ (varying from $100 \mathrm{keV} / \mathrm{amu}$ to $10 \mathrm{MeV} / \mathrm{amu}$ ) ealculated according to the procedure described in paper 1. These charge states are estimated to be accurate upto $10 \%$. Compared to the dimension 10-50 A.U. of the heliosphere it can then be concluded that these charge states are still preserved in the interplanetary medium in the vicinity of the

Table 2. Average equilibrium values $i$ of charged states of ions in interstellar space (composed mainly of hydrogen) (taken from Durgạprasad and Biswas 1977)

\begin{tabular}{ccccc}
\hline & \multicolumn{4}{c}{ Kinetic energy (eV/amu) } \\
\cline { 2 - 5 } $\begin{array}{c}\text { Atomic } \\
\text { number }\end{array}$ & $10^{6}$ & $5 \times 10^{5}$ & $10^{6}$ & $10^{7}$ \\
\hline 6 & 1.8 & 4.3 & 5.4 & 6 \\
7 & 1.8 & 4.7 & 5.9 & 7 \\
8 & 1.9 & 5.0 & 6.4 & 8 \\
10 & 2.1 & 5.7 & 7.3 & 10 \\
12 & 2.3 & 6.2 & 8.2 & 12 \\
14 & 2.5 & 6.7 & 8.9 & 14 \\
26 & 3.3 & 8.8 & 12.5 & 25 \\
\hline
\end{tabular}


earth. Even after acceleration to about $10 \mathrm{MeV} / \mathrm{amu}$ from $0.1 \mathrm{MeV} / \mathrm{amu}$, the charge states given in table 2 represent the values of $i$ in the interplanetary medium.

As regards modulation in interplanetary medium, it is noted that ions having energies around $10 \mathrm{MeV} / \mathrm{amu}$ and in partly ionized states will be modulated in the region 50 to 1 A.U. quite differently than the ions of fully stripped nuclei with the same energy/amu. This is because the magnetic rigidity, $R$, of an ion is given by $R=(A P c / i)$, and the lesser the value of $i / A$, the greater is the rigidity of the particle for the same value of $P c$. The oxygen and neon ions have higher rigidities than the carbon ions and will be hence less modulated. Thus oxygen and neon ions are relatively enhanced in the inner solar system.

The differential modulation of $\mathrm{C}, \mathrm{O}, \mathrm{Ne}, \mathrm{Mg}$ and $\mathrm{Si}$ ions have been calculated as described in paper 1. For this purpose, the calculations of Garcia-Munoz et al (1975) are taken to be representative of the modulation for the 1973-74 period. Also using the 'force-field' solution of Gleeson and Axford (1968) it was estimated that for this period the force-field potential is around $270 \mathrm{MeV} /$ amu. Using these data, the differential modulation of oxygen and carbon ions is estimated to be a factor of 1.3. The relative modulation factors of $\mathrm{He}, \mathrm{N}, \mathrm{Ne}, \mathrm{Mg}, \mathrm{Si}$ and $\mathrm{Fe}$ ions with respect to carbon ions thus calculated are $0 \cdot 5,1 \cdot 2,1 \cdot 4,1 \cdot 5,1 \cdot 6$ and $2 \cdot 2$ respectively. In this paper, it is shown that during propagation in interstellar space, the charge states are preserved as these are equilibrium values and hence the ions can travel fairly long paths in interstellar space.

\subsection{Acceleration process}

The ions with equilibrium charge states having kinetic energies about $100 \mathrm{keV} / \mathrm{amu}$ were assumed, in paper 1 , to be acceletated at the heliospheric boundary. On the basis of recent astronomical observations it seems more likely that interstellar shock fronts would be capable of accelerating these ions from $100 \mathrm{keV} / \mathrm{amu}$ to the energy range of 1 to $50 \mathrm{MeV} / \mathrm{amu}$ quite efficiently. This is discussed in more detail in the recent model of the stellar origin of the anomalous cosmic rays as given later.

\subsection{Propagation in interplanetary space}

Next we examine the question, what are the charge states of these ions in interplanetary space? It is shown in paper 1 that the mean free path for stripping of these ions (e.g. C, O) in interplanetary space are $2 \times 10^{4} \mathrm{~A}$. U. and $3 \times 10^{5} \mathrm{~A}$. U. at energies of $10^{5} \mathrm{eV}$ and $10^{7} \mathrm{eV}$ respectively. These values are several orders of magnitude higher compared to the dimensions of about $50 \mathrm{~A}$. U. of the heliosphere. It can then be concluded that these charge states are preserved in interplanetary medium and in the vicinity of $1 \mathrm{~A} . \mathrm{U}$.

\subsection{Relative abundances}

In table 1, the demodulated abundances are compared to the abundance values for novae DQ Her 1934 obtained by absorption method by Antipova (1974), who observed that abundances of $\mathrm{C}, \mathrm{N}, \mathrm{O}$ were abnormal as compared to those of normal stars and of solar system. Abundance in another novae HR DEL 1967 was also 
abnormal, but oxygen was very depleted, as given in paper 1. From these observaions, it is clear that novae abundances show, peculiarities as well as variations. Emission processes and interstellar acceleration mechanism are also expected to modify the abundances of the ions accelerated to $\sim 10 \mathrm{MeV} / \mathrm{amu}$. It can thus be concluded from table 1 that peculiarities of the $\mathrm{He}, \mathrm{C}, \mathrm{N}, \mathrm{O}$, Ne abundances observed in the anomalous component of low energy cosmic rays could partly exist in astrophysical objects such as novae.

\section{Stellar model of O-type stars with strong stellar wind as source of low energy cosmic rays}

As discussed in the last section, it was suggested in paper 1 that novae type objects having peculiar composition may act as the sources of injecting particles. While this possibility exists, a number of new astrophysical results have been obtained in recent years which provide new clues on the origin of low energy cosmic rays from stellar sources. The new model of Biswas et al (1981) is based on these recent observations.

In this model, it has been proposed that O-type stars with strong stellar winds act as the source of injection of low energy cosmic rays. These ions, of energies 10-

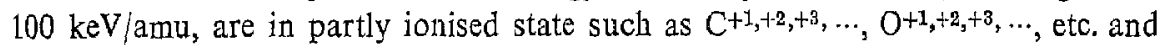
hence these have very low rate of Coulombian energy loss in hot, thin interstellar plasma of $T \approx 5 \cdot 10^{5} \mathrm{~K}$ and $N_{c} \approx N_{p} \approx 3 \times 10^{-3} \mathrm{~cm}^{-3}$ which fills $70-80 \%$ of instellar medium (see e.g. Melrose 1979). As a result the ions can travel fairly long distances in interstellar medium. During interstellar propagation a fraction of the ions will interact with shock fronts of supernovae remuants and gain energy by Fermi mechanism. Several authors (Blanford and Ostriker 1978; Ostriker 1979) have recently worked out the efficient mechanism of acceleration of galactic cosmic rays in interstellar shock fronts. It is suggested that low energy ions can be accelerated to 1-50 MeV/amu energy range in such shock fronts in interstellar medium and thus low energy cosmic rays are generated. These cosmic ray ions enter the heliosphere from interstellar medium and are channelled by the interstellar magnetic field and large scale magnetic field in the heliosphere (Nagashima and Morishita 1979, 1980). The general outline of the model of the stellar origin of low energy cosmic rays is given in figure 1 . We shall briefly discuss some significant aspects of this model.

\subsection{O-type stars}

It is now well established that $O$-type stars with $20 \mathrm{M}_{\odot}<\mathrm{M}<100 \mathrm{M}_{\odot}$ manifest very high mass loss rates (see Conti and Deloore 1979; Conti and McCray 1980). The mass loss occurs in the form of very strong stellar winds and these account for high rate of mass loss of $3 \cdot 10^{-6} \mathrm{M}_{\odot}$ per year. This can be compared to the normal mass loss of the Sun of $10^{-14} \mathrm{M}_{\odot}$ per year in the form of solar wind. In the spectral classification $O$-type stars have the highest surface temperature of $T>30,000^{\circ} \mathrm{K}$ and the largest masses in the main sequence phase. The luminosities of 0 -type stars are very high which are on the average about $10^{38}$ ergs sec $^{-1}$ i.e. $\sim 10^{0} \mathrm{~L}_{\odot}$ These 
Figure 1. Model of the origin of low energy cosmic rays from stellar wind (Biswas et al 1981)

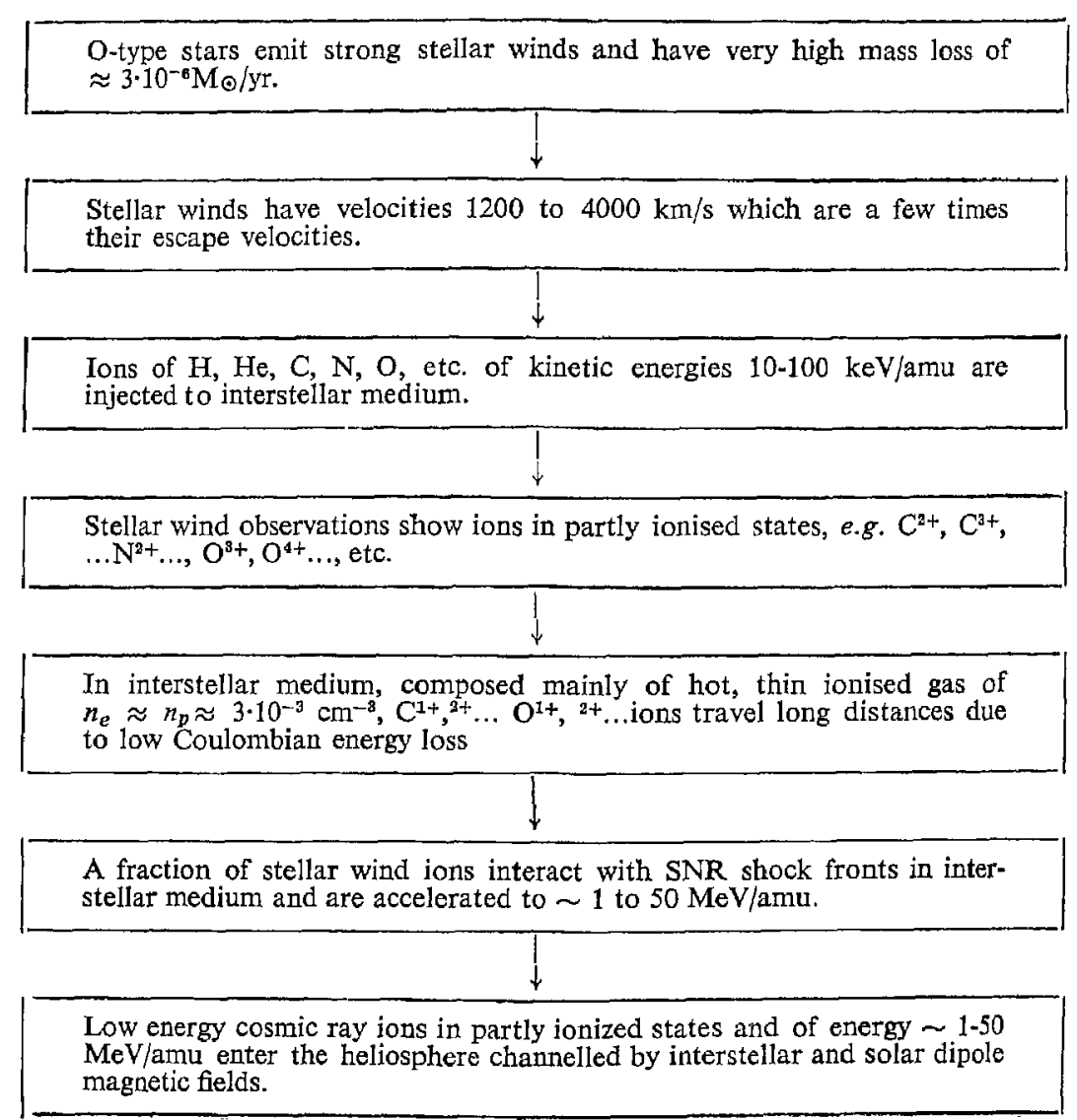

stars consequently have short life time of $\sim 10^{6}$ years. As a result $O$-type stars are rare and there are about $3 \times 10^{5}$ such stars in the galaxy, i.e. a fraction of $10^{-6}$ of the total number of stars. Because of the short lifetime they have gone through about $10^{4}$ generations during the lifetime of the galaxy. Their high mass loss rate contributes to a large fraction of new matter injected into the interstellar space.

The terminal velocities of stellar winds from O-type stars range from 1200 to 4000 $\mathrm{km} / \mathrm{sec}$ and these are typically a few times their escape velocities. These velocities correspond to ion energies of $10-100 \mathrm{keV} / \mathrm{amu}$. Because of very high velocities exceeding the escape velocities, these ions are considered in the present model as good candidate for the source of injection of low energy cosmic rays.

\subsection{Ion states and propagation in interstellar medium}

Observational data show that ions of the stellar wind are injected in the interstellar medium in partly ionized state such as $\mathrm{C}^{+2},+3 \ldots, \mathrm{N}^{+2},{ }^{+3} \ldots, \mathrm{O}^{+2},+3 \ldots$. It seems likely that such elements exist in many ion states. The recent observation of interstellar medium has dramatically changed our picture of the interstellar medium. 
From soft $x$-ray and interstellar ultraviolet studies it is now indicated that a large fraction (70-80\%) of interstellar medium is flled with ionized gas of high temperature, $T \approx 5 \cdot 10^{5} \mathrm{~K}$ and of low density $n_{e} \approx n_{p} \approx 3 \times 10^{-3} \mathrm{~cm}^{-3}$ (see e.g. Melrose 1979). It is estimated that repeated SN explosions maintain the interstellar medium at high temperature.

We have calculated the energy loss of the partly ionized elements of stellar wind in the hot, thin interstellar medium using rigorous equations of Coulombian energy loss of ion in two component plasma according to Butler and Buckingham (1962). From these calculations it is noted that low energy ions such as $\mathrm{He}^{+1}, \mathrm{C}^{+2}$, etc. with initial energy of about $100 \mathrm{keV} / \mathrm{amu}$ can travel a long distance in hot, thin interstellar medium till their energies are reduced to $\sim 10 \mathrm{keV} / \mathrm{amu}$. This arises from the decreasing energy loss rate with decreasing energy beyond the electron loss maximum. In case of $\mathrm{He}^{+1}$ ion this path length is calculated as $4 \times 10^{20} \mathrm{~cm}$. For other elements, the path lengths and the distances travelled would depend on their ionization states and these are expected to be within one order of magnitude of the above.

\subsection{Acceleration in interstellar medium}

It has been calculated by several authors that supernovae remnant shock fronts can travel a large distance in the hot and low density interstellar medium (see e.g. Ostriker 1979). The interaction of fast ions with the expanding shock fronts can provide efficient acceleration process through first order of Fermi process. A number of authors have worked out such processes for acceleration of high energy cosmic rays (e.g. Ostriker 1979). In this work we propose that nonrelativistic heavy ions can be accelerated from 10 to $100 \mathrm{keV} / \mathrm{amu}$ range to 1 to $50 \mathrm{MeV} / \mathrm{amu}$ by means of collisions with SNR shock fronts in interstellar medium. In this case, the energy gain requirements are of the order of a factor of $5 \times 10^{2}$. Acceleration efficiency will be dependent on the ion states of the particles and this effect is probably responsible for unusual abundance ratios such as $\mathrm{O} / \mathrm{C}$. Further calculations on these aspects of the model are in progress.

\subsection{Entry into heliosphere}

The accelerated heavy ions would then enter the heliosphere and give rise to the observed flux of low energy cosmic ray ions. Depending on the polarity of the sun and the large scale magnetic field direction of the interplanetary mganetic field and the direction of interstellar magnetic field, these cosmic ray ions would be channelled favourably or not, as discussed by Nagashima and Morishita $(1979,1980)$. Thus the long term variation of the intensity of the low energy cosmic rays may be understood.

\section{Concluding remarks}

The above model provides a suitable framework in which various properties of low energy cosmic rays may be understood. Detailed calculations of the processes involved are in progress, with a view to obtain more insight into the phenomena relating to the origin of low energy cosmic rays. 


\section{References}

Antipova L I 1974 Highlights of astronony, ed. G Contopoulos, (Dordrecht: D Reidel) p. 501

Biswas S and Durgaprasad N 1980 Space Sci. Rev. 25285

Biswas S, Durgaprasad N, Nevatia J, Venkatavaradan V S, Goswami J N, Jayanthi U B, Lal D and Mattoo S K 1975 Astrophys. Space Sci. 33337

Biswas S, Durgaprasad N and Trivedi Sushma S 1981 Proc. 17th Int. Cosmic Ray Conf. Paris 2 (Saclay: CENS) p. 314

Blanford R D and Ostriker J P 1978 Astrophys. J. Lett. 221 L229

Butler S T and Buckingham M J 1962 Phys. Rev. 1261

Chan J H and Price P B 1974 Astrophys. J. 190 L39

Conti P S and Deloore C W H (ed) 1979 IAU Symp. No. 83 (Dordrecht: D Reidel)

Conti P S and McCray R 1980 Nature (London) 2089

Durgaprasad N 1977 Astrophys. Space Sci. 47435

Dutgaprasad N and Biswas S 1977 Proc. 15th Int. Cosmic Ray Conf. Plovdiv 2 (Plovdiv: Bulgarian Acad. Sci.) p. 103

Fisk L A, Kozlovsky B and Ramaty R 1974 Astrophys. J. 190 L35

Gleeson L J and Axford W I 1968 Astrophys. J. 1541011

Garcia Munoz M, Mason G M and Simpson J A 1975 Astrophys. J. 202265

Hovestadt D, Klecker B, Gloeckler G, Ipavich F M, Fan C Y and Fisk L A 1979 Proc. 16th Int. Cosmic Ray Conf. Kyoto 3 (Japan: Univ. of Tokyo) p. 255

Hovestadt D, Vollmer O, Gloeckler G and Fan C Y 1973 Phys. Rev. Lett. 31 650; and Proc. 13th Int. Cosmic Ray Conf. Denver 2 (Colorado: Univ. of Denver) p. 1498

McDonald F B, Teegarden B J, Trainor J H and Webber W R 1974 Astrophys. J. 187 L105

McKibben R B, Pyle K R and Simpson J A 1979 Astrophys. J. 227 L 147

Melrose G B 1979 Plasma Astrophys. Vol. 1. (New York: Gordon and Breach) p. 20

Mewaldt R A, Stone E C, Vider S B and Vogt R E 1975 Proc. 14th Int. Cosmic Ray Conf. Munich 2 (Max Planck Instiut, Munich), p. 798

Nagashima G and Morishita I 1979 Proc. I6th Int. Cosmic Ray Conf. Kyoto 3 (Japan: Univ. of Tokyo), p. 325

Nagashima G and Morishita I 1980 Planet. Space Sci. 28195

Ostriker J P 1979 Proc. 16th Int. Cosmic Ray Conf. Kyoto 2 (Tokyo: Univ. of Tokyo), p. 124

Webber W R 1979 Proc. 16th Int. Cosmic Ray Conf. Kyoto 14 (Tokyo: Univ. of Tokyo), p. 271

Webber W R, Damle S V and Kish J 1972 Astrophys. Space Sci. 15245

Webber W R, McDonald F B, Trainor J H, Teegarden B J and Von Rossenvinge T T 1975 Proc. I4th Int. Cosmic Ray Conf. 12 (Munich: Max Planck Institut) p. 4233 